\title{
Do researchers have their heads in the clouds, bogged down with scientific medical detail? [art therapy maybe a simpler alternative]
}

\begin{abstract}
This art work depicts a woman with her head in the clouds. Researchers can at times have their heads in the clouds, bogged down in theoretical details related to medication options when art therapy may offer solutions with benefits outweighing that of medication. Not dissimilar to the idiom wood for the trees definition where someone doesn't see the wood(s) made by the grouping of the trees, as they are concentrating on individual trees so can't get a general understanding of a situation because they are concentrating on detail. People with diverse medical illnesses at times maybe shuffled to different GPs or specialists, prescribed medications, with variable likelihood of effectiveness or adverse event possibilities and hence outcomes maybe on a continuum of positive to negative. Clinical trials monitor objective and subjective outcomes over time. This scientific way of treating illness is generally accepted, but maybe over medicalized, complicated and burdensome with information overload, multiple prescriptions, forms, documents, appointments, time and cost commitments. Alternatively, people with mental health disorders [trauma], other psychological disorders, loss of memory, early dementia, or post stroke may benefit from art therapy and research evidence in relation to art therapy effectiveness in these realms mentioned report benefit.
\end{abstract}

Volume 5 Issue I - 2020

\author{
Deborah Joy Hilton \\ Deborah Hilton Statistics Online, Australia
}

Correspondence: Deborah Joy Hilton, Deborah Hilton Statistics Online, Melbourne,Victoria,Australia, Tel + 6|3983085|4, Email deborah.hilton@gmail.com

Received: January 02, 2020 | Published: February 17, 2020

Keywords: art therapy, complementary therapies, therapeutics, diagnosis

\section{Introduction}

Previously an abstract on this topic was presented at the Australian Society for Medical Research conference which was themed; Inspired creativity; when art meets science. ${ }^{1}$ The poster and rapidfire presentation displayed an art work done by the author's daughter [Miss Emma Hilton] whom is an emerging artist depicting a woman with her head in the clouds, ${ }^{2}$ Figure 1. It is possible that researchers can at times have their heads in the clouds, getting bogged down in theoretical details related to medication or referral options when simple art therapy may in fact offer solutions with benefits that far outweigh that of medication. Not dissimilar to the idiom wood for the trees definition where someone doesn't see the wood (s) made by the grouping of the trees, because they are concentrating on the individual trees so can't get a general understanding of a situation because they are concentrating on detail.

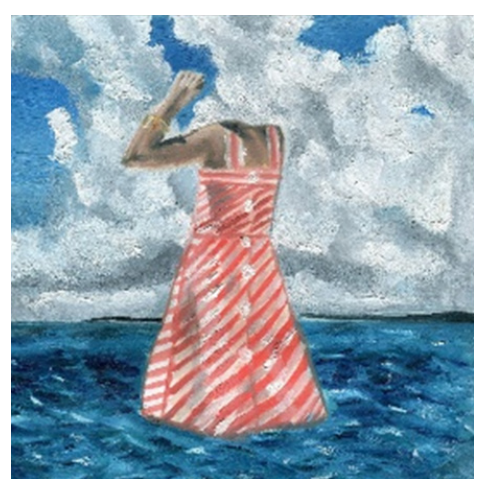

Figure I Woman with her head in the clouds [Reproduction of an art work painted by Miss Emma Hilton].

\section{Methods}

In order to show the complexities related to the process of diagnostic decision making several examples from the literature are detailed below.

Diagnostic decision making is a complex process and is used to rule out a plethora of medical conditions that a patient may possibly have. It is important before sending people through the tangled web of diagnostic rooms within a hospital facility or medical care centre that reaching a diagnosis that relates to a person's symptoms and signs will improve outcomes otherwise the process of labelling is somewhat superficial and superfluous. In addition, the process can prove costly, time consuming and emotionally over whelming if there is no treatment for a condition once diagnosed. Alternatively, if there are treatment options; a correct, comprehensive and accurate diagnostic pathway is crucial. Once a person has been diagnosed with a medical illness, then they enter the health system, and those with diverse medical illnesses at times maybe shuffled to different GPs or specialists, prescribed medications that have variable likelihood of effectiveness or adverse event possibilities and hence outcomes maybe on a continuum of positive to negative. Persons may be invited to join clinical trials whereby they may be monitored and followed up with objective and subjective outcomes determined over time. This scientific way of treating illness is generally accepted, yet can become over medicalized, at times complicated and burdensome in terms of either information overload, multiple prescriptions, forms, documents, appointments, time and cost commitments.

Several examples below show the difficulties related to diagnostic and treatment options. Figure 2 below is a diagnostic decision tree for the classification of mental health and developmental disorders of infancy and early childhood. This shows the complexity of differentiation, and categorisation. ${ }^{3}$ 


\section{DIagnOStic Classification 03: DeCISION TREe - AXISI (PRIMARY DIagnOSIS)}

Answer all the questions on this tree ( 1 to 9 ). Two primary conditions are possible, but rare. Use "Rule Out" when you need more information in making a differential diagnosis

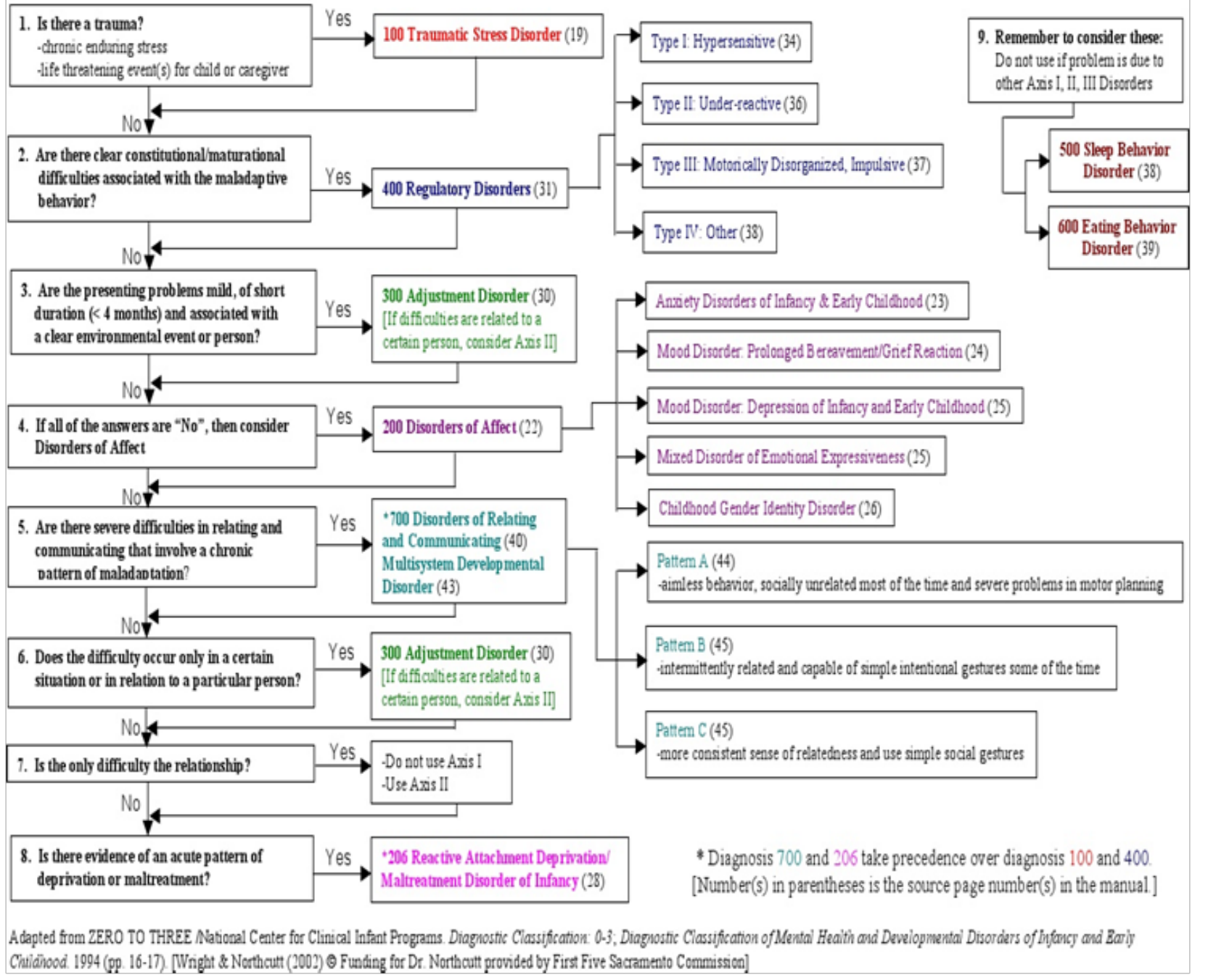

Figure 2 The diagnostic decision tree for the classification of mental health and developmental disorders of infancy and early childhood [Wright and Northcutt 2005].

Psychcentral.com is a website that describes symptoms and treatments of mental health disorders. They list many disorders and the list is vast and varied. ${ }^{4}$

If you just consider the common adult mental health disorders, some are listed below;

Alcohol/Substance Abuse

Alcohol/Substance Dependence

Anxiety Disorders

Adult Attention Deficit/Hyperactivity Disorder

Bipolar Disorder

Depression
Eating Disorders

Generalized Anxiety Disorder

Obsessive-Compulsive Disorder

Opioid Use Disorder Symptoms

Panic Disorder

Postpartum Depression

Posttraumatic Stress Disorder

Schizophrenia

Seasonal Affective Disorder

Social Anxiety Phobia 
Obviously if someone is having symptoms of distress, it could be quite difficult and confusing going through the maze of diagnostic tests, in order to determine which of the above is relevant. This could become overwhelming and distressing. In addition, for some of these situations, a diagnosis requires a cut point, in that for conditions such as anxiety or depression where everyone in the community can at times have symptoms, then a diagnosis is just a word. If someone is depressed as they have lost a loved one or someone is anxious because of job stress, then a diagnosis may not change or alter the situation. In fact, art therapy maybe a better investment of time and energy rather than going through this diagnostic maze, as it may offer relief of symptoms associated with the depression or anxiety, apart from being labelled as having a mental illness, so that it in fact alleviates some of the distress or apathy.

Another example is Figure 3 below that shows the complexities of diagnosis, as it relates to insomnia and sleep disorders. ${ }^{5}$ Again, while a diagnosis is important to rule out pathology or sinister reasons for signs or symptoms, it should also be remembered that simple art therapy may offer relief of anxiety or depression that can contribute to conditions like insomnia. For some people they may prefer to try this alternative option firstly to see if there is benefit, in particular if they feel a stressor maybe the cause before embarking upon a series of tests to rule out other reasons for symptoms or signs. This maybe a far easier pathway to travel. Obviously if a person has symptoms that indicate more serious issues then just depression or anxiety as a cause, for example they are stopping breathing during sleep which may indicate apnea then testing and diagnosis should be the first line of action, but if just stressors related to job, work or school are apparent and maybe suggestive of cause, then art therapy maybe tried initially to assess benefit possibilities before a convoluted, rabbit warren series of investigations begin in the search for other reasons.

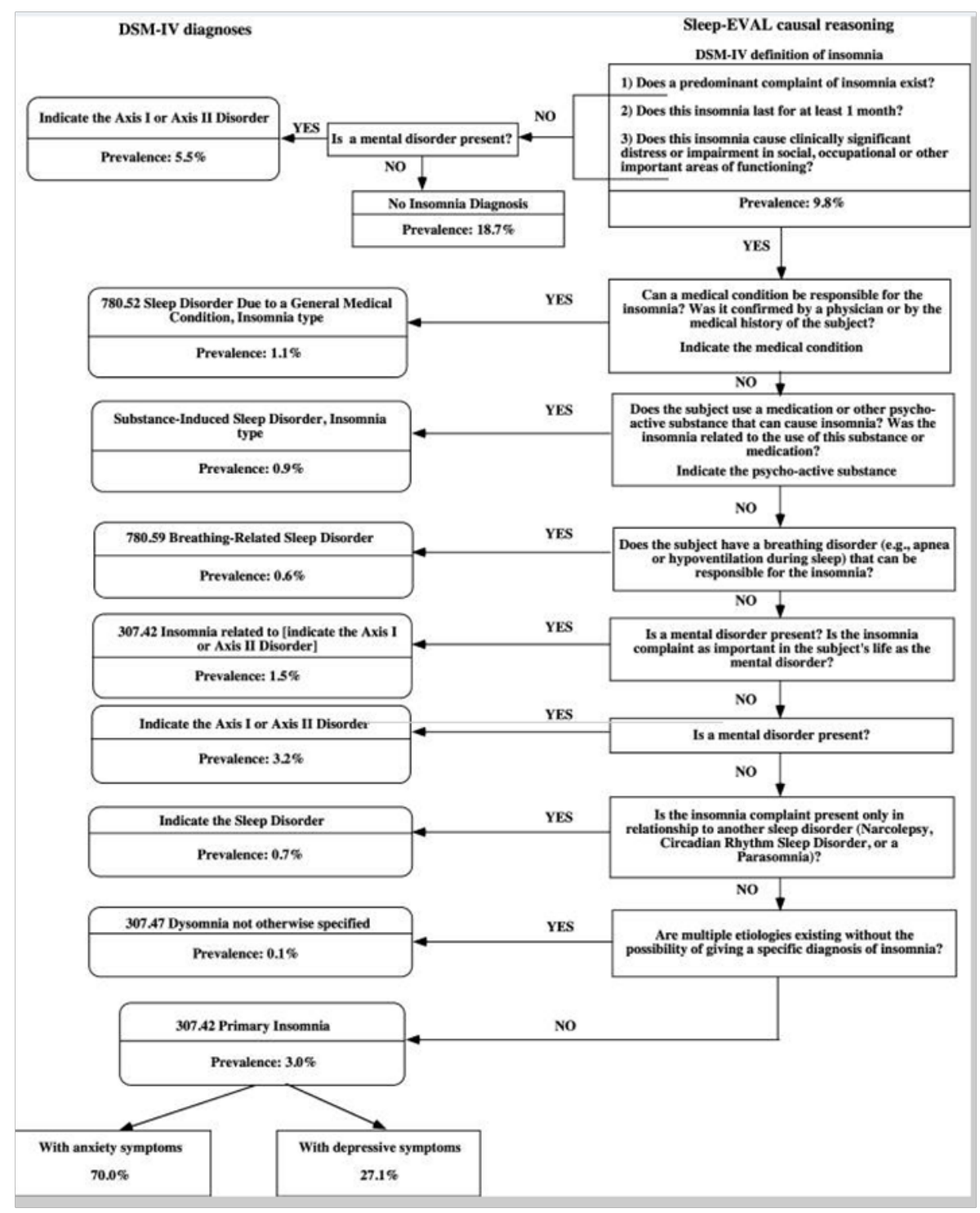

Figure 3 Epidemiological and clinical relevance of insomnia diagnosis algorithms according to the DSM-IV and the International Classification of Sleep Disorders (ICSD). Sleep Medicine 2009; 10:952-60. Ohayon MM and Reynolds CF. Epidemiological and clinical relevance of insomnia diagnosis algorithms according to the DSM-IV and the International Classification of Sleep Disorders (ICSD). Sleep Medicine 2009; 10: 952-60. 
The Qld Health Department, have prepared a document titled; Guidelines for Care and Referral of Adults with Type 2 Diabetes. ${ }^{6}$ Tables within section two of this document discuss assessment criteria and this can include a range of examinations and tests.

Assessment of diabetes status can include; symptoms, investigations such as [urine, blood pressure, renal function, lipids, liver function tests], waist circumference, weight, body mass index, personal and family history. Diaries of smoking, nutrition, physical activity and alcohol intake can also be recorded. Other assessment includes; self-care, home blood glucose monitoring, foot risk assessment and examination, eyes, psychological status and blood glucose. Other plans include registration with the national diabetes services scheme, medication review, setting goals, setting management plans, discussing cultural issues, team care arrangements and notification to the driving licence authority.

Section 3 within this document discusses referral options and necessities. Referral options for diabetes care can include professionals such as; primary care coordinator, general practitioner, diabetes educator, indigenous health worker, remote area health nurse, dietician, podiatrist, ophthalmologist, optometrist, psychologist, endocrinologist, nephrologist, neurologist, cardiologist, vascular specialist, exercise professional/ physiotherapist and/or pharmacist.

Oviously for conditions such as diabetes whereby medication maybe necessary to control blood glucose levels, proper assessment and referral is critical. Art therapy in these instances maybe an adjunct, but not an alternative and mention of one study whereby subjects whom had diabetes were included in an art therapy class is given in the section below tited; art therapy.

Figure 4 shows a chart for the suggested treatment of posttraumatic stress disorder in emergency service workers. This is downloaded from the black dog institute website. Treatment after identification of symptoms and signs involves cognitive behaviour therapy, eye movement desensitisation and reprocessing therapy, or selective serotonin reuptake inhibitors.?

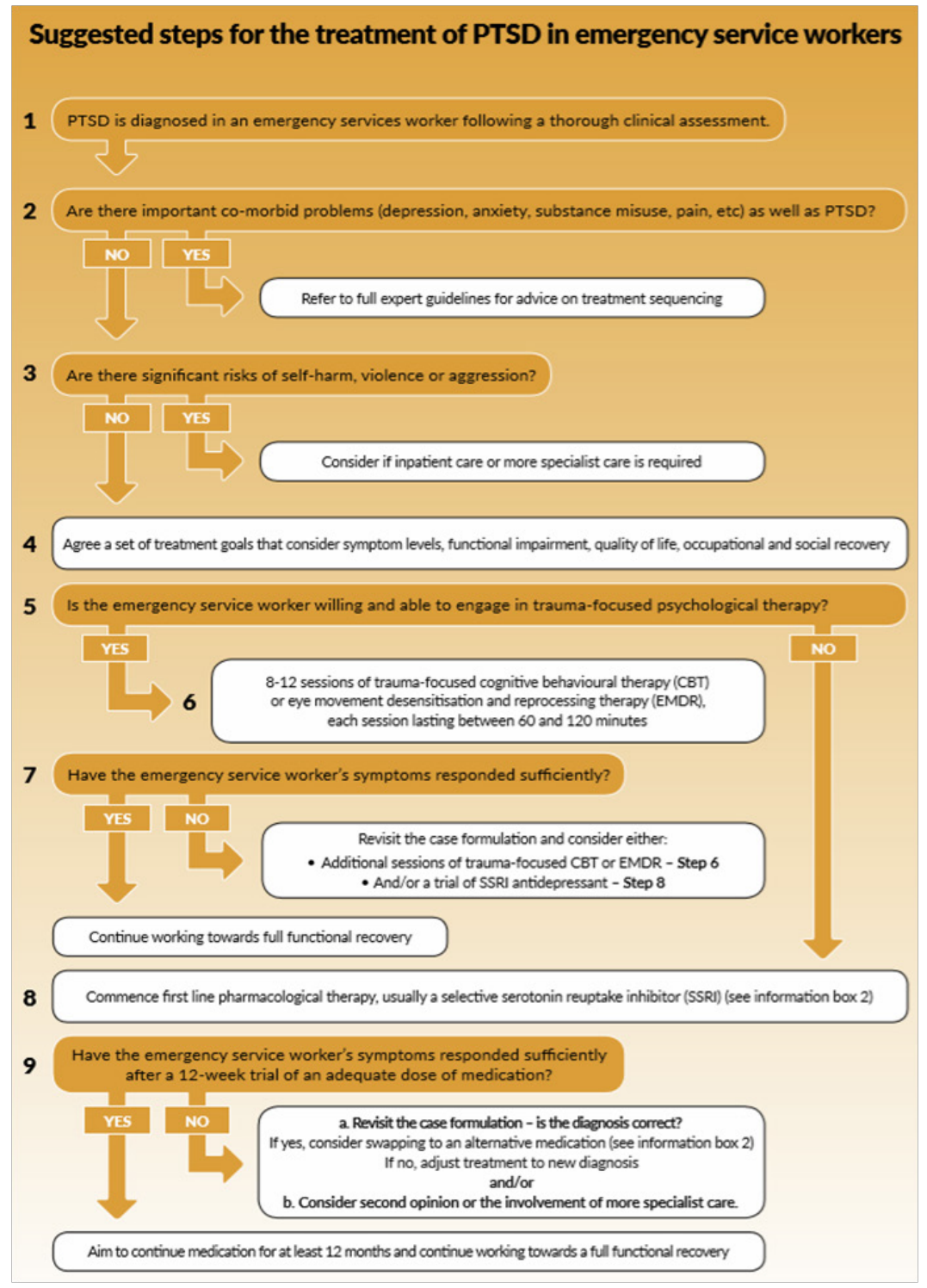

Figure 4 Treatment of post-traumatic stress disorder [Black Dog Institute - blackdoginstitute.org.au]. 
Again, the diagnostic decision tree is quite complex and detailed and in fact for stress, art therapy may improve the symptoms of mental health disorders and the evidence for this will be detailed more fully in the next section.

Art therapy options provide a relaxed, creative, fun and social alternative that may appeal to some people. Not only are there benefits in that these options may be less cumbersome in terms of attending, time and cost both being considered, but in addition there are research studies or clinical trials that show positive benefit and results and these are detailed below.

\section{Results}

A literature review including systematic review and clinical trial evidence related to art therapy effectiveness and benefit was done and is detailed below.

There are numerous clinical research studies that report art therapy benefit as it relates to a number of clinical objective and subjective outcomes. The results of these manuscripts are detailed below.

1. In people with psychosis, a review identified 18 papers and of these, the high-quality qualitative articles reported that therapists and clients considered art therapy to be a useful, valuable, meaningful, and satisfactory intervention. These results were based on a small number of studies. ${ }^{8}$

2. The Burma art therapy program evaluation assessed the effects of art therapy for healing the effects of trauma among refugee youth. Thirty participants were included at baseline and follow up, being given four validated clinical assessment tools measuring symptoms of depression, anxiety and behavioural problems. This evaluation showed some effects of art therapy with benefits in the area of anxiety and self-concept. ${ }^{9}$

3. Eum and Yim reported that literature and art therapy can identify the emotional status of patients and serve as a useful auxiliary tool to help stroke patients in their rehabilitation process. ${ }^{10}$ Via art works, a patient's mental problems, internal conflicts, emotions, and psychological status can undergo a healing process.

4. Case studies and several small trials suggest that art therapy has benefit for Alzheimer's disease and other dementias. Therapy can engage attention, offers pleasure, improves neuropsychiatric symptoms, social interactions, and self-confidence. ${ }^{11}$

5. Uttley and colleagues performed a meta-analysis that included eleven randomised controlled trials (533 patients). ${ }^{12}$ This systematic review and cost-effectiveness analysis reported that the limited available evidence was that art therapy was associated with positive effects compared with control in a number of studies in patients with different clinical profiles being non-psychotic mental health disorders. In 7 of the 11 studies, art therapy was associated with significant positive changes in mental health symptoms in those persons having art therapy when compared to the control group.

6. A study assessing the addition of intensive art therapy in youth with poorly controlled type 1 diabetes mellitus, included $n=29$ participants [intervention group $n=16$, control group $n=13$ ]. Results were a decrease in hemoglobin Alc in the intervention group compared with control so in essence it may improve glycaemic control. ${ }^{13}$
7. To examine the effects of art and music therapy on depression and cognitive function of the elderly $[n=94] .{ }^{14}$ Art therapy revealed a statistically significant difference between before and after treatment in terms of improving participant's depression.

Hence the above references demonstrate that art therapy has been shown to assist those with mental health disorders [trauma, psychosis, non-psychotic mental health disorders], the elderly, people with diabetes, stroke sufferers, or those with Alzheimer's or other dementias. Obviously as previously mentioned, a proper diagnosis and expert treatment is crucial in many instances whereby if someone doesn't seek treatment, the person's condition may deteriorate or they may suffer worsening health status. However, in cases where there is clearly a cause for symptoms or signs that may just relate or contribute to stress or anxiety which precipitates depression, insomnia or other clinical manifestations, then art therapy may be considered as an adjunct therapy or even in some cases an alternative therapy. There is clearly a place and value for the healing effects of artistic expression.

\section{Discussion}

\section{Art therapy exhibitions and programs}

The author has attended the black dog exhibition ${ }^{15}$ for a number of years, not for therapy, but due to the fact she receives invitations from various art groups as a result of her daughter's artist talent with one of her art works shown in Figure 5. ${ }^{15}$

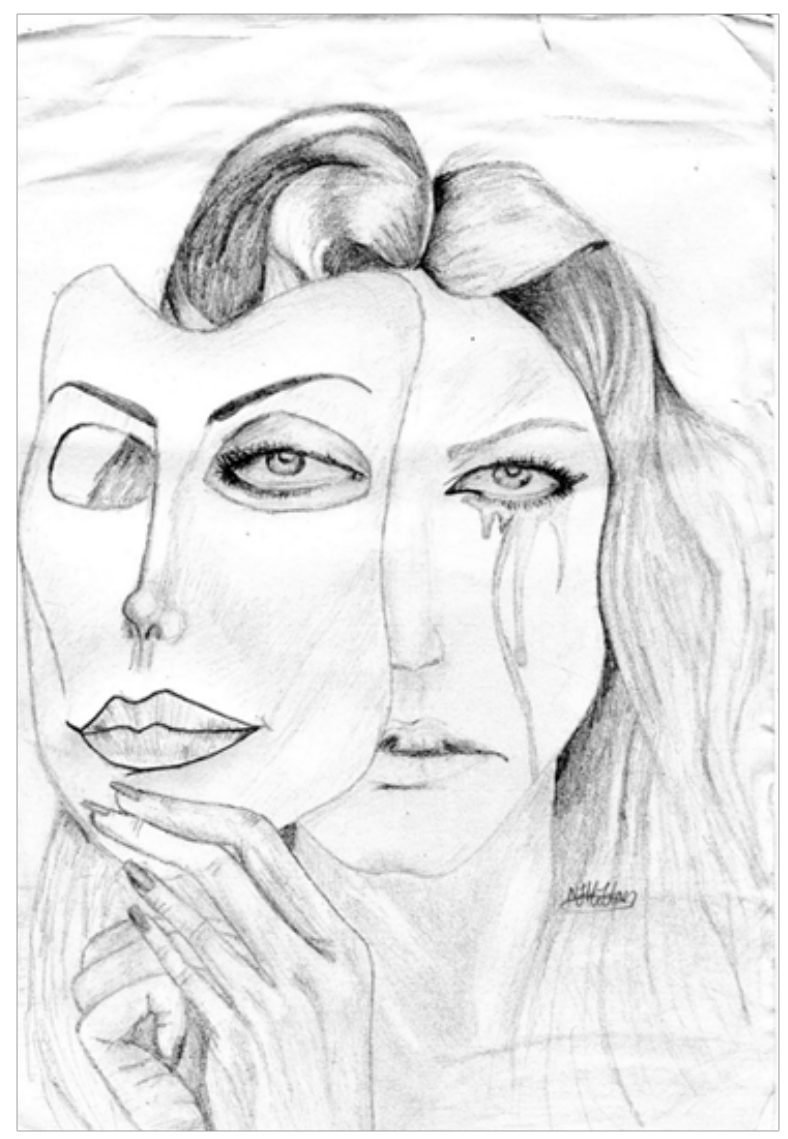

Figure 5 An art work by Miss Natasha Hilton submitted to a black dog art show.

Other works that are featured on the 'The black dog community art project', facebook page can be viewed on this publicly available URL. 
https://www.facebook.com/BlackDogArtExhibition/?tn-str=k*F [accessed 26/3/2018]. ${ }^{15}$

Various artists use their skill and talent aside from painting lovely portraits, but also to help people via the art therapy domain or to detail their experiences by painting art works that portray their plight.

David Bromley emerged as a painter in the mid-1980s and has exhibited worldwide including Australia. ${ }^{16,17}$

He was one of a number of artists, whom were included in the Art Program at Sydney Children's Hospital, Randwick [Sydney Children's Hospitals Foundation] ${ }^{18}$ This program aims to use art as a healing tool and offers a refuge from the intense emotions associated with illness, giving them a way to express their feelings and allowing them respite from what they are going through. His work is included in the art program more specifically his works are in the collection that comprises approximately 900 artworks.

\section{A war artist}

In October 2011, the Australian War Memorial commissioned Ben Quilty as the official war artist to visit Afghanistan with the Australian Army [Jan Murphy Gallery. ${ }^{17,19}$ After Afghanistan, is a soft cover book providing an insight into the work generated from this experience. Other publications detailing the artist's works are also available from this gallery, including those which detail the plight of Syrian refugees and their forsaken homeland. As an official war artist in Afghanistan his role was to interpret the experiences of Australian Defence Force personnel participating in Operation Slipper. ${ }^{19}$

\section{Conclusion}

In the words of 'Thomas Merton' "Art enables us to find ourselves and lose ourselves at the same time." In the words of the author of this manuscript 'Pour paint colours onto a palette, mix your colours and you never know your life may become colourful, vibrant and swirly. Art can be messy, but then so can be life for many people so it is what you make of the mess that counts, and creating a masterpiece is not out of the realms of possibility if you give it a go!'

\section{Funding}

None.

\section{Acknowledgments}

Miss Emma Hilton, Miss Natasha Hilton [amateur artists].

\section{Conflicts of interest}

The author declares no conflicts of interest.

\section{References}

1. Hilton DJ. Australian society for medical research. Inspired creativity. When art meets science. Adelaide. Do researchers have their heads in the clouds, bogged down with scientific medical detail? [art therapy maybe a simpler alternative]. Poster and rapid-fire presentation. 2018.

2. Hilton D. Whitehorse leader-your news. Box Hill High School Archibox Award. 2012.

3. Wright C, Northcutt C. Adapted from ZERO TO THREE. Diagnostic classification of mental health and developmental disorders of infancy and early childhood: Revised edition (DC:0-3R). Washington. 2005.

4. Psych Central [Symptoms \& Treatments of Mental Disorders].

5. Ohayon, MM, Reynolds CF. Epidemiological and clinical relevance of insomnia diagnosis algorithms according to the DSM-IV and the International Classification of Sleep Disorders (ICSD). Sleep Medicine. 2009;10:952-960.

6. Qld Health Department (2006). [Guidelines for Care and Referral of Adults with Type 2 Diabetes].

7. Black Dog Institute

8. Attard A, Larkin, M. Art therapy for people with psychosis: a narrative review of the literature. Lancet Psychiatry. 2016;3(11): 1067-1078.

9. Rowe C, Watson-Ormond R, English L, et al. Evaluating art therapy to heal the effects of trauma among refugee youth: the burma art therapy program evaluation. Health Promot Pract. 2017;18:26-33.

10. Eum Y, Yim J. Literature and art therapy in post-stroke psychological disorders. Tohoku J Exp Med. 2015;235(1):17-23.

11. Chancellor B, Duncan A, Chatterjee A. Art therapy for Alzheimer's disease and other dementias. $J$ Alzheimers Dis. 2014;39(1):1-11.

12. Uttley L, Stevenson M, Scope A, et al. The clinical and cost effectiveness of group art therapy for people with non-psychotic mental health disorders: a systematic review and cost-effectiveness analysis. $B M C$ Psychiatry. 2015;15:151.

13. Harel S, Yanai L, Brooks R, et al. The contribution of art therapy in poorly controlled youth with type 1 diabetes mellitus. J Pediatr Endocrinol Metab. 2013;26(7-8):669-673.

14. Im ML, Lee JI. Effects of art and music therapy on depression and cognitive function of the elderly. Technol Health Care. 2014; 22:453458.

15. The black dog community art project facebook page.

16. Anthea Polson Art [David Bromley CV].

17. Jan Murphy Gallery [Artists- Ben Quilty]

18. Sydney Children's Hospitals Foundation [Art Program].

19. Australian War Memorial [Ben Quilty Official War Artist - Press Release 2011. Ben Quilty: after Afghanistan - biography]. 